\title{
Search for neutrino emission from extended sources with the IceCube detector
}

\author{
The IceCube Collaboration ${ }^{1}$, \\ ${ }^{1}$ http://icecube.wisc.edu/collaboration/authors/icrc15_icecube \\ E-mail: epinat@icecube.wisc.edu
}

\begin{abstract}
The IceCube Neutrino Observatory, a cubic kilometer telescope located in the Antarctic ice, offers unique opportunities to study high-energy neutrino emission from galactic and extragalactic sources. The Galactic plane is the brightest source of gamma rays in the sky, and it is believed to be also one of the brightest sources of very energetic neutrinos. The first discovery of an astrophysical high-energy neutrino flux has recently been announced by the IceCube collaboration and, although no clear sources have been found so far, it is reasonable to investigate whether a Galactic component might be contributing to the observed flux. As indicated by the HESS gamma-ray survey and by Milagro as well, many of the sources populating the Galactic plane are in fact extended sources. The sensitivity and discovery potential of IceCube for neutrinos coming from extended regions using 6 years of data are being presented in this contribution.
\end{abstract}

Corresponding authors: Elisa Pinat ${ }^{* \dagger}$, Juan Antonio Aguilar Sánchez ${ }^{\dagger}$,

${ }^{\dagger}$ Université Libre de Bruxelles,

Interuniversity Institute for High Energies (IIHE), 1050 Brussels, Belgium

The 34th International Cosmic Ray Conference,

30 July- 6 August, 2015

The Hague, The Netherlands

*Speaker. 


\section{Introduction}

IceCube is a cubic kilometer neutrino detector installed in the ice at the geographic South Pole between depths of $1450 \mathrm{~m}$ and $2450 \mathrm{~m}$ [1]. Detector construction started in 2005 and finished in 2010. During the construction period, IceCube collected data in four different configurations, until the final 86 string one was achieved starting from 2011. Neutrino reconstruction relies on the optical detection of Cherenkov radiation emitted by secondary particles produced in neutrino interactions in the surrounding ice or the nearby bedrock. Neutrinos have unique properties that can be used to probe various astrophysical processes. Produced in the same environment as Cosmic Rays (CRs) and Gamma Rays, their neutral charge allows them to travel straight from the source to Earth, preserving directional information. Furthermore, the fact that they only interact weakly allows them to travel large distances without being hindered by intervening matter. Astrophysical neutrinos are also tracers of hadronic interactions, and the identification of these neutrino sources may help to clarify cosmic ray acceleration processes. The recent discovery by IceCube of a diffuse high-energy astrophysical neutrino flux [2][3] is now established at a $7 \sigma$ confidence level [4], but no sources have been identified yet. Finding neutrino sources in the sky requires locating an excess of events from a particular direction over the background of atmospheric neutrinos and muons. The energy distribution of events is used as discriminator between the signal and the background hypotheses [5]. In these proceedings we focus on spatially extended sources: many have been already identified in the gamma sky and if a neutrino counterpart is found they will help unravel the mysteries of cosmic ray acceleration. Sensitivities and discovery potential will be shown on data from three years of operation in partial levels of completion (IC-40, IC-59 and IC-79) and the first three years of the completed 86 string detector (IC86-I, IC86-II, IC86-III). The previous extended source analysis was limited to the data of the first IC86-I sample, and the results are shown in [6].

\section{Scientific motivation}

The Galactic plane is the brightest source of $\gamma$-rays in the sky [7][8]. Among other processes, the $\gamma$-ray emission from the Galaxy originates also from the cosmic ray interaction in the interstellar medium. In these interactions, the production of neutral and charged pions, and their subsequent decay, results into $\gamma$-ray, electron/positron and neutrino emission. The HESS survey of the inner part of the Galactic Plane [9] has revealed a number of bright extended $\gamma$-ray sources and it is thus the $\gamma$-ray astronomy that provides a strong motivation to look for extended sources. The same sources are also seen in the survey of the Galactic Plane above $100 \mathrm{GeV}$ performed by FermiLAT [10], and it is possible that these are also the locations of recent injections of $E>1 \mathrm{TeV}$ cosmic rays in the Galaxy [10]. If the observed $\gamma$-rays are produced by CR interactions, a very-high-energy neutrino flux should be associated as well. If detected, this would represent an unambiguous proof of the hadronic nature of the sources.

Motivation in favour of a dedicated extended source analysis is also obtained from Fig. 1. Assuming the presence of extended sources and modelling the signal injection accordingly to its extension, this plot shows the comparison between an analysis that uses the regular point source method to analyse this signal and an analysis that instead takes into account the spatial extension 
in the likelihood method, assuming each time a different spatial extension from $1^{\circ}$ to $5^{\circ}[6]$. The median angular resolution of the detector varies with energy, but for well reconstructed tracks can be $<1^{\circ}$. The discovery potential for an $\mathrm{E}^{-2}$ flux of the regular point source analysis method is compared to the one calculated with our extended source analysis. In this case, the same value used to simulate the spatial extension enters the likelihood calculation, showing that, when the correct source extension is used, the improvement is noticeable. Since the source extension is not known a priori, several assumptions will be made, from one to five degrees.

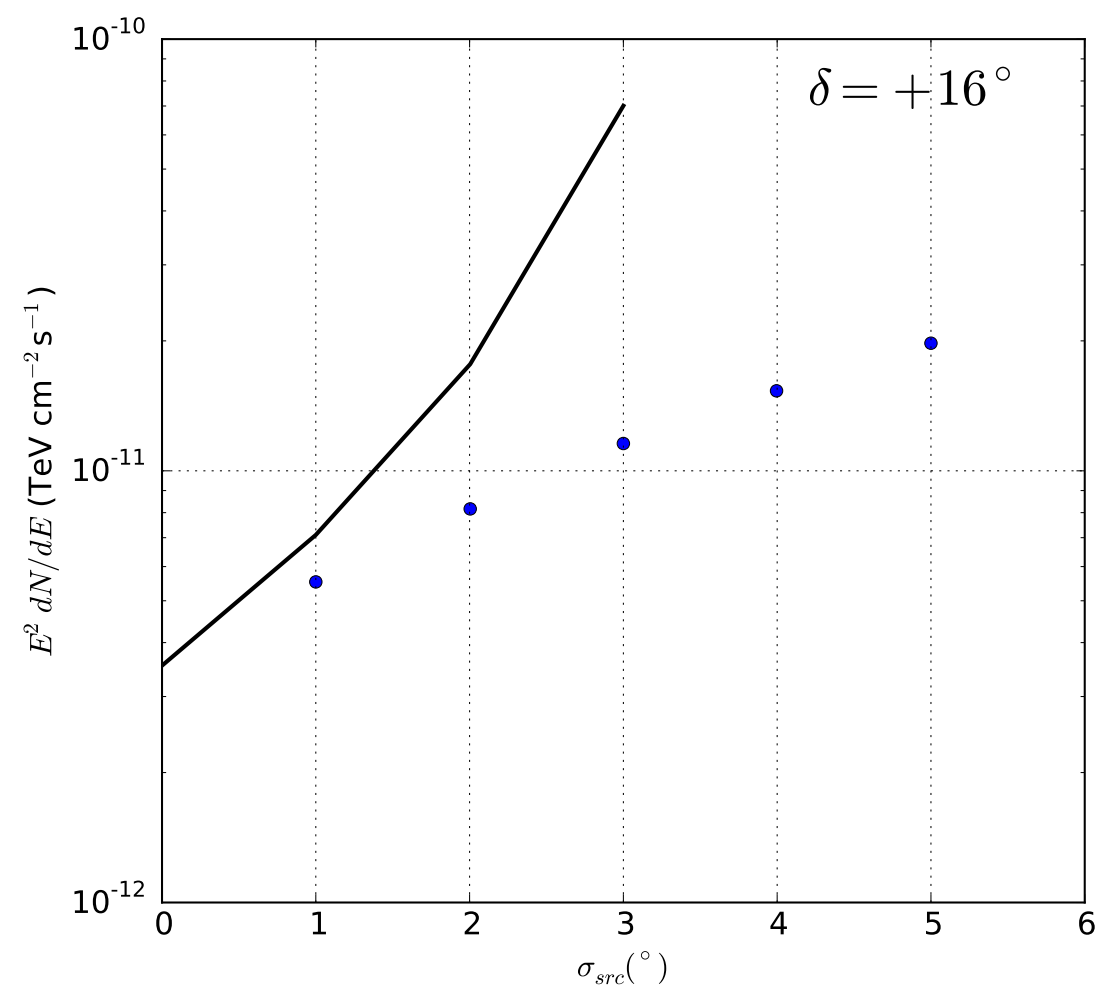

Figure 1: Discovery potential for an $\mathrm{E}^{-2}$ flux from an extended source at a declination of $16^{\circ}$ for one year of IC86 data with no trial factor correction. The regular point source analysis (solid-line) is compared to the discovery potential of the extended source analysis using always the correct source extension (blue dots).

\section{Analysis method}

This analysis uses an unbinned maximum likelihood test [11], that allows to calculate the significance of an excess of neutrinos above the background for a given direction. Both the reconstructed direction of the event and the reconstructed deposited muon energy are used to discriminate between signal and background. Since signal events have a harder energy spectrum compared to 
the atmospheric neutrino and muon background [12], this method performs better than a simple directional clustering approach [13]. The signal and background probability density functions (p.d.f.) are a function of the reconstructed declination and the reconstructed muon energy. For a data sample of $N$ total events, the p.d.f. of the $i^{t h}$ event in the $j^{\text {th }}$ sample (IC40, IC59, IC79, IC86-I, IC86-II or IC86-III) with reconstructed energy $E_{i}$ and angular distance to the source $\left|\vec{x}_{i}-\vec{x}_{s}\right|$ is given by:

$$
P_{i}^{j}\left(\left|\vec{x}_{i}-\vec{x}_{s}\right|, E_{i}, \gamma, n_{s}^{j}\right)=\frac{n_{s}^{j}}{N^{j}} S_{i}^{j}+\left(1-\frac{n_{s}^{j}}{N^{j}}\right) B_{i}^{j}
$$

where $S_{i}^{j}$ and $B_{i}^{j}$ are the signal and background p.d.f. respectively, and $n_{s}^{j}$ is the number of signal events expected from the corresponding $j^{\text {th }}$ sample. The signal p.d.f. used is given by:

$$
S_{i}^{j}=s_{i}^{j}\left(\left|\vec{x}_{i}-\vec{x}_{s}\right|, \sigma_{i}, \sigma_{s r c}\right) \varepsilon_{i}^{j}\left(E_{i}, \delta_{i}, \gamma\right)
$$

here $s_{i}^{j}$ is the space contribution and depends on the angular uncertainty of the event, $\sigma_{i}$, the angular difference between the reconstructed direction of the event and the source position and the extension of the source. The spatial probability distribution function is still assumed to be a 2-D Gaussian but, contrary to the regular point source analysis, the width is determined by:

$$
S_{i}^{j}=\frac{1}{2 \pi\left(\sigma_{i}^{2}+\sigma_{s r c}^{2}\right)} e^{-\frac{\left|\vec{x}_{i}-\vec{x}_{s}\right|^{2}}{2\left(\sigma_{i}^{2}+\sigma_{s c}^{2}\right)}}
$$

The energy $\varepsilon_{i}^{j}$ and background $B_{i}^{j}$ p.d.f remain the same as for the regular point source method described in Ref. [3]. The simulation of an extended source is performed by sampling events from several injected point sources distributed according to the source extension. To parametrize the extended source we use the Kent distribution [14], which for small values of sigma $\left(<8^{\circ}\right)$ can roughly be considered as a Gaussian normalized on a sphere. The signal injection simulation is used for sensitivity and discovery potential calculations, but also to calculate the relative efficiencies of the different detector geometries when combining several years of data.

The likelihood is a function of two fit parameters: $n_{s}$, the number of signal events originating from the source, and $\gamma$, the spectral index of a neutrino source with a power law spectrum, which are maximized for a given location in the sky. The significance is then estimated using the loglikelihood ratio as the test statistic as described in Ref. [11]. This proposed analysis is expanded to several years of different detector geometries as described in Ref. [15].

\section{Performance}

In this section we show the performance of an all-sky scan searching for sources of $1^{\circ}, 2^{\circ}, 3^{\circ}$, $4^{\circ}$ and $5^{\circ}$ extension as a function of declination. Figure 2 shows the sensitivity for a $v_{\mu}$ flux at the 90\% C.L. whereas Fig. 3 shows the discovery potential. The silver band represents the coverage from $1^{\circ}$ to $5^{\circ}$ source extension for six years of data, whereas the black lines are relative to the previous 4 years analysis, the solid line for $1^{\circ}$ extension and the dotted one for $5^{\circ}$. For comparison, the four years regular point source analysis reports, for an $\mathrm{E}^{-2}$ spectrum, a median sensitivity value at $90 \%$ C.L. of $\sim 10^{-12} \mathrm{TeV}^{-1} \mathrm{~cm}^{-2} \mathrm{~s}^{-1}$ for energies between $1 \mathrm{TeV}-1 \mathrm{PeV}$ in the northern sky and of $\sim 10^{-11} \mathrm{TeV}^{-1} \mathrm{~cm}^{-2} \mathrm{~s}^{-1}$ for energies between $100 \mathrm{TeV}-100 \mathrm{PeV}$ in the southern sky [6]. For the 
moment, six years of data have been simulated by adding two more times the dataset relative to IC86-I. These datasets are the ones that have been used for point source searches, where the background rejection is optimised for better angular resolution. For this reason, sensitivity studies will be performed to determine instead how to optimize the event selection for exended sources.

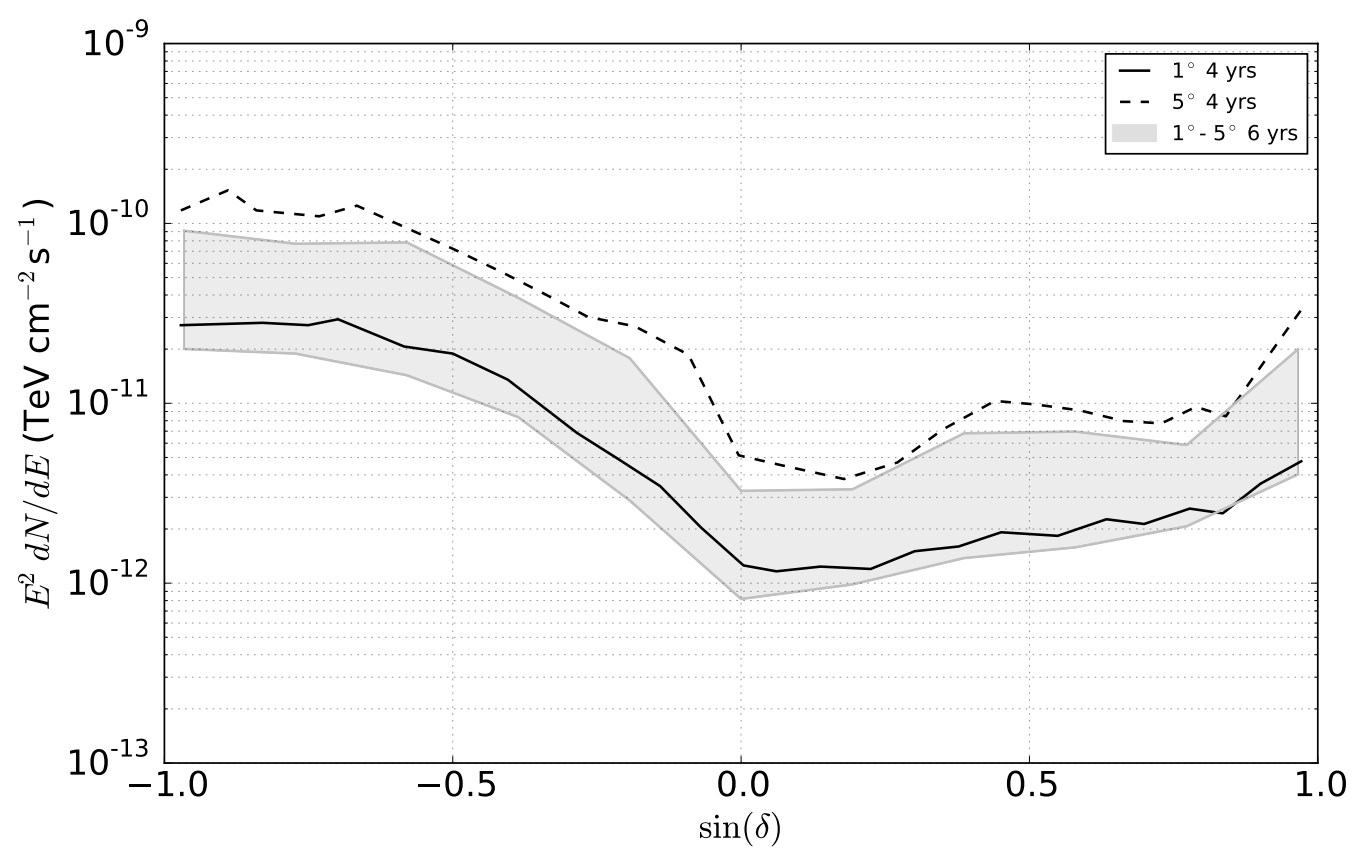

Figure 2: Sensitivity for an $\mathrm{E}^{-2}$ neutrino flux from an extended source considering various extensions from one to five degrees, using the correct extension in the likelihood. The silver band represent the 6 years sensitivity interval from $1^{\circ}$ to $5^{\circ}$ source extension, whereas the black solid and dotted lines represent the sensitivity for $1^{\circ}$ and $5^{\circ}$ for 4 years of data.

\section{Conclusions and Outlook}

The goal of this analysis is to produce a set of significance skymaps for various source extensions. For each extension scan, the highest significant coordinate will be selected and the most significant location of the various maps will be used to determine the final p-value. The trial factor arising from the all-sky scan of each search will be taken into account by generating average background samples using the right ascension scrambling technique in order to estimate how often the background can produce a p-value as significant as the one observed [5]. The trial factor among the various skymaps will be conservatively assumed to be just the number of produced maps.

Although not as sensitive as regular point source searches for point-like sources, extended searches investigate a different signal hypothesis where the no-extension assumption is strongly penalized if the neutrino source has truly an extension. Many astrophysical surveys have already 


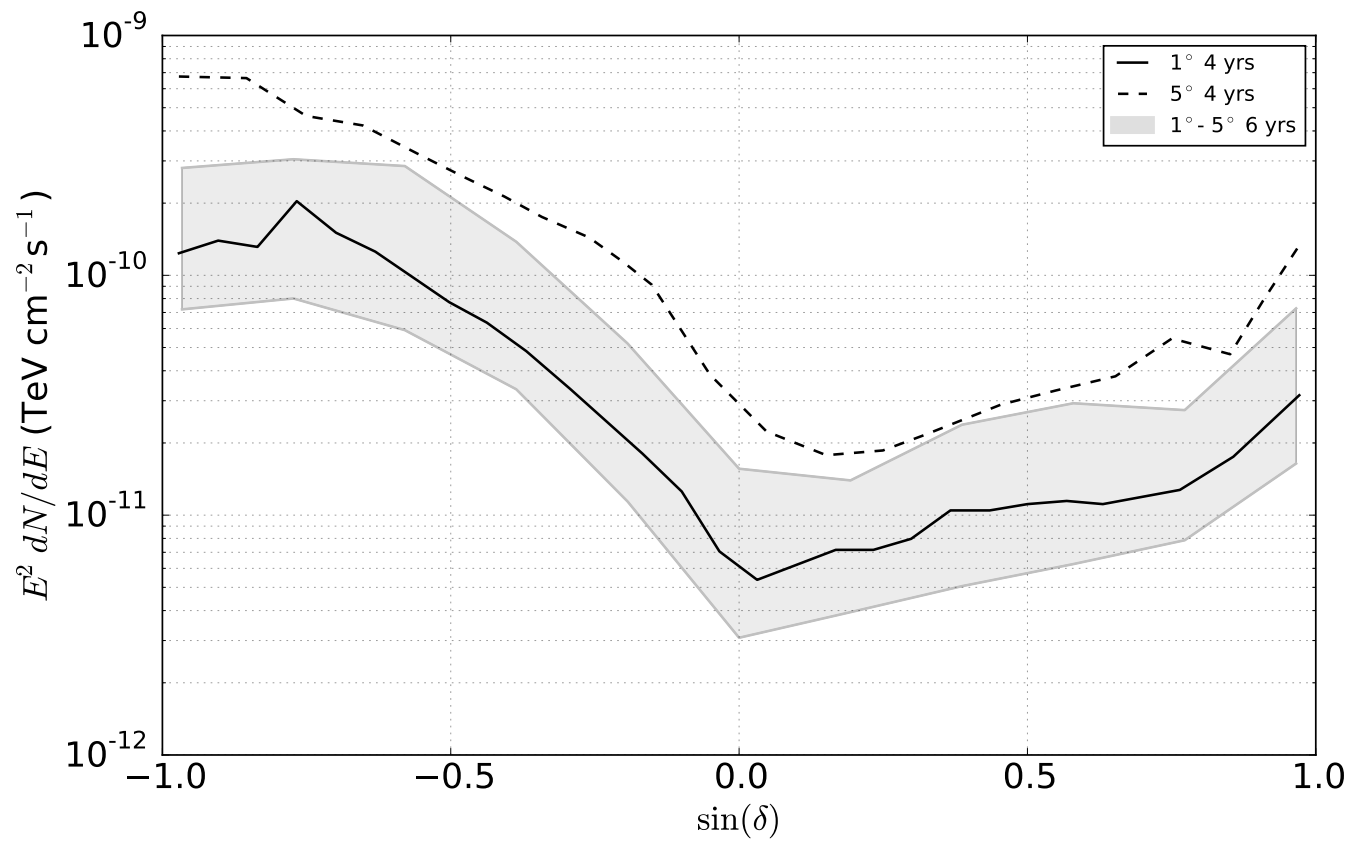

Figure 3: Discovery potential for an $\mathrm{E}^{-2}$ neutrino flux from an extended source considering various extensions from one to five degrees, using the correct extension in the likelihood. The silver band represent the 6 years discovery potential interval from $1^{\circ}$ to $5^{\circ}$ source extension, whereas the black solid and dotted lines represent the discovery potential for $1^{\circ}$ and $5^{\circ}$ for 4 years of data.

revealed the presence of extended sources in the gamma sky, but the identification of a neutrino counterpart is still a challenge. If found, however, it would help reveal the sources and underlying physics processes of cosmic ray acceleration. 


\section{References}

[1] IceCube Collaboration, A. Achterberg et al., Astroparticle Physics 26 (2006) 155-173, [astro-ph/0604450].

[2] IceCube Collaboration, M. Aartsen et al., Science 342 (2013) 1242856, [arXiv: 1311.5238 ].

[3] IceCube Collaboration, M. Aartsen et al., Phys.Rev.Lett. 113 (2014) 101101, [arXiv: 1405.5303 ].

[4] IceCube Collaboration, PoS(ICRC2015)1081, these proceedings (2015).

[5] J. Braun, J. Dumm, F. De Palma, C. Finley, A. Karle, et al., Astroparticle Physics 29 (2008) 299-305, [arXiv:0801.1604].

[6] IceCube Collaboration, M. Aartsen et al., arXiv:1406.6757.

[7] W. L. Kraushaar, G. W. Clark, G. P. Garmire, R. Borken, P. Higbie, V. Leong, and T. Thorsos, The Astrophysical Journal 177 (Nov., 1972) 341.

[8] M. Ackermann et al., The Astrophysical Journal 750 (May, 2012) 3, [arXiv: 1202.4039 ].

[9] F. Aharonian et al., Astronomy and Astrophysics 477 (Jan., 2008) 353-363, [arXi v : 0712 . 1173 ].

[10] A. Neronov and D. V. Semikoz, Phys. Rev. D 85 (Apr, 2012) 083008.

[11] J. Braun, M. Baker, J. Dumm, C. Finley, A. Karle, and T. Montaruli, Astroparticle Physics 33 (Apr., 2010) 175-181, [arXiv:0912.1572].

[12] IceCube Collaboration, M. Aartsen et al., Phys.Rev. D91 (2015), no. 2 022001, [arXiv:1410.1749].

[13] IceCube Collaboration, R. Abbasi et al., The Astrophysical Journal 732 (2011), no. 118.

[14] J. T. Kent, Journal of the Royal Statistical Society. Series B (Methodological) (1982) 71-80.

[15] IceCube Collaboration, M. Aartsen et al., The Astrophysical Journal 779 (2013), no. 2132. 